\title{
ENFERMERÍA COMO PROFESIÓN: EN BUSCA DE NUEVAS PREGUNTAS ${ }^{1}$
}

\author{
NURSING AS PROFESSION: \\ SEARCHING NEW QUESTIONS
}

\author{
RiCARDO A. Ayala
}

\begin{abstract}
RESUMEN
Durante su desarrollo evolutivo, la enfermería ha sido abordada como una actividad ocupacional basada en una vocación por un fin público, cuyo rol se ha transformado en una de las claves de las organizaciones de salud modernas. Pero ante todo, los esfuerzos se han enfocado en las prerrogativas de estatus profesional. La construcción de estas prerrogativas, no obstante, se han basado primariamente en los aspectos estructurales de la enfermería, vale decir, respondiendo a cómo la ocupación de enfermería se estructura para desarrollar su rol ocupacional, pero no a cómo interactúa con el resto del sistema ocupacional en que se desenvuelve, la ecología en que sostiene relaciones recíprocas con otros miembros del sistema. En este artículo planteo la necesidad de una propuesta teórica que apoye el desarrollo profesional y la construcción de estatus desde una aproximación conceptual sistémica.
\end{abstract}

Palabras clave: Enfermería, división del trabajo, ocupaciones, sociología.

\begin{abstract}
During their evolutionary development, nursing has been considered as an occupational activity based on a vocation for a public purpose and whose role has become central in modern health organisations. But above all, nurses' efforts have been focused on a prerogative of full professional status. Such prerogative construction, however, seems to rest on structural concerns alone, instead of functional concerns. By this is meant, the question seems to head into how nursing is structured to undertake its work, instead of how nursing interacts with the rest of the occupational system with which it has reciprocal relationships. This paper highlights the necessity of a conceptual framework that provides a theoretical proposal to support nursing development from a comprehensive and systemic approach.
\end{abstract}

Key words: Nursing, labour division, occupations, sociology.

Fecha recepción: 17/07/12 Fecha aceptación: 25/03/13

\footnotetext{
${ }^{1}$ Este artículo emerge en contexto de la investigación doctoral: “Towards a Sociology of Nursing as an Occupation: Conceptual Dilemma for a Discipline searching for Profession".

* Enfermero. PhD® en Sociología. Departamento de Sociología, Ghent Unibersity, Bélgica. Email: RicardoALexis. AyalaValenzuela@UGent.be
} 


\section{INTRODUCCIÓN}

El estatus profesional de enfermería ha sido una cuestión fervorosamente debatido durante las últimas cuatro décadas, debate en que el principal foco de interés se ha concentrado en responder si la enfermería califica o no como profesión. El concepto de profesión, sin embargo, es impreciso y confuso, debido a que puede ser empleado de formas diferentes e incluso contradictorias. Profesión, por ejemplo, puede referirse a un concepto técnico que refleja cierto nivel de experticia, pero este concepto también es empleado como atributo o símbolo social, una construcción que refiere a un estatus honorífico. No obstante, todos estos sentidos del concepto parecen ser un estado deseable de una actividad ocupacional y, tomando en consideración la deseabilidad de este estado, asumiré en este análisis que la enfermería desea ser reconocida como una profesión.

Estudiar las profesiones puede tomar diferentes perspectivas o enfoques para profundizar la discusión en las distintas direcciones del concepto. En la bibliografía reciente el carácter profesional de la enfermería es dado por sentado como una característica autoevidente, generalmente argüido en base a aspectos estructurales, es decir, argumentando un conjunto de características que virtualmente definen una profesión.

Tal argumento, en general desarrollado por especialistas de salud, no puede ser considerado como un análisis exhaustivo en lo que concierne a las profesiones, y por tanto debe enfrentar muchas limitaciones. Por ejemplo, algunas características de este enfoque son el conocimiento disciplinar propio, la autonomía y el monopolio de su jurisdicción ocupacional. Es aceptado que el cuerpo de conocimientos que la enfermería ha desarrollado ha madurado exponencialmente y que, por tanto, ello refleja su naturaleza profesional; pero por otro lado puede haber legítimos desacuerdos respecto a lo relacio- nado con la autonomía de su ejercicio práctico, y también respecto al monopolio de tal ejercicio. Esta discordancia entre distintos criterios del enfoque estructural en efecto refleja tanto debilidades en la formulación de las prerrogativas profesionales, como la inconsistencia del enfoque en sí mismo para concluir si una ocupación califica o no como profesión, y es lo que tomo como punto inicial en mi argumento: se necesitan marcos teóricos a través de los cuales las prerrogativas profesionales puedan ser perfeccionadas y fortalecidas.

Por otra parte, y en el centro del análisis que traigo al debate, ha de considerarse que las ocupaciones están organizadas socialmente. Esto significa que hay modos sociales de interconexión entre diferentes actividades de un mismo ámbito de trabajo, pues las instituciones en tanto organizaciones reproducen en gran medida las características de la sociedad en que funcionan. Esto da como consecuencia, por ejemplo, que las ocupaciones operan en una lógica jerárquica y que se ubican dentro de un sistema ocupacional en que distintos mecanismos de control definen los límites de la práctica y, por ende, el trabajo profesional. Más que una lista de características que en teoría pueden definir una profesión, el análisis necesita comenzar con preguntas tales como ¿cómo, a quién, con quién o con qué una ocupación hace lo que hace? Interrogantes como éstas antagonizan con la pregunta clásica "¿es la enfermería una profesión?", tradicionalmente respondida, una vez más, en base a una lista de criterios o requisitos. Perceptiblemente, la noción de sistema no ha sido introducida en la investigación de enfermería en tanto profesión, a pesar de ofrecer una amplia gama de perspectivas analíticas, las cuales subrayan la interconexión inseparable de las esferas de la vida social, como son la competición, la clase social, el estatus, la movilidad social y las inequidades sociales. Éste es el vacío que intentaré fundamentar en este artículo, aunque no pretendo sea un marco de trabajo final, 
sino un planteamiento teórico que debe ser sustancialmente contrastado con indagación empírica.

En primer lugar, comenzaré desarrollando una visión general de la aproximación tradicional, la cual ha sido ampliamente aplicada en el análisis del trabajo profesional en el contexto de la enfermería en las sociedades industriales. Luego discutiré cómo la aproximación tradicional se fundamenta en una noción teológica del orden social y cómo ha influido en la estructuración de los grupos profesionales incluyendo el desarrollo de la enfermería. Hacia el final analizaré el principal problema, focalizando en cómo las limitaciones de un análisis estructural justifican la necesidad de un planteamiento sistémico para la ocupación de enfermería y las implicancias en su desarrollo profesional.

\section{Industrialización y estructura profesional}

La discusión sobre las profesiones y el trabajo profesional ha sido materia de gran debate en las sociedades industriales. Como esfera relevante de la vida en sociedad, la sociología ha tomado parte en el debate sobre el trabajo y en cómo ciertas ocupaciones devienen profesiones. Flexner (1) fue el primer autor en plantear una visión sobre el trabajo profesional, influyendo hasta el punto que varios escritos posteriores siguieron sus observaciones sobre el trabajo profesional del médico. Por ejemplo, Richmond(2) incorporó la lógica de la medicina "observación-diagnóstico-tratamiento" al trabajo social; o Cook (3) quien trajo los estándares de Flexner de la educación superior a las escuelas de derecho, así como Le Rosignol (4) a la economía, Morrill (5) al periodismo, y los Bixler $(6,7)$ junto a Leddy y Pepper (8) a la enfermería.

Basado en observaciones del comportamiento profesional, el trabajo de Flexner se convirtió en $e l$ enfoque de trabajo profesional en las sociedades industriales, conocido como enfoque estructural o enfoque tradicional, compuesto por un conjunto de rasgos característicos que supuestamente definen una profesión. La transformación causada por la transición de una economía agraria a una economía industrial subrayó la importancia central del trabajo en la vida en sociedad, por lo que surgieron muchas preguntas sobre las ocupaciones y especialmente sobre cómo unas habrían evolucionado hacia un estatus profesional.

La noción de actividad intelectual fue planteada por Flexner como el primer rasgo de un profesional; la naturaleza de la actividad profesional es el proceso de pensamiento en la resolución discrecional de problemas complejos de la existencia humana. En sus propias palabras, "una inteligencia libre, ingeniosa, y sin obstáculos aplicada a problemas, y el esfuerzo por entenderlos y dominarlos" (1). No obstante, el mero intelectualismo no podría tener lugar en el campo de las profesiones, pues el objeto práctico dentro de un campo definido es el rasgo medular de ese conocimiento.

Además, la consecución de fines sociales, enfatizada por Flexner como un pilar profesional, sitúa a los profesionales en una posición moral que los involucra con los intereses públicos por sobre sus propios intereses. Este sentido de rectitud se entiende garantizado a través de una organización profesional.

Por otro lado, sobre la base de una educación selectiva y autorregulada, obtienen una considerable formación, la cual finalmente termina creando una fraternidad. De esta manera toda la vida profesional y personal tiende a organizarse en torno a ciertos temas de interés: tópicos de conversación, lenguaje, maneras de vestir, preferencias de viajes, tipo de lectura, y en definitiva la creación de determinadas formas de gusto (9). Lo que Flexner enfatiza aquí es crucial para ilustrar mi análisis: a) la fraternidad que conforman las profesiones es en otras palabras una casta; b) su vida personal se ubica dentro de una clase profesional; c) los profesionales que son conscientes de su estatus tienen "una conciencia de clase"; y d) la actividad profesional 
garantiza un salario digno y un alto nivel de vida en recompensa.

Este fenómeno de estratificación en el trabajo es, para mí, una idea particularmente clara en los escritos de Flexner. Por ejemplo, es evidente el modo en el que el trabajo de la enfermera y del farmacéutico en relación con el médico se basa en la subordinación y la obediencia, aspectos que en modo alguno llegan a ser objeto de cuestionamiento o crítica.

Sobre el farmacéutico, Flexner (1) sostiene:

"El médico piensa, decide y ordena. El farmacéutico obedece. Por supuesto, obedece discrecionalmente, con inteligencia y habilidad, pero en esencia obedece. Por tanto, el farmacéutico es un brazo de la profesión médica, una forma especial y distinta de oficio superior, no una profesión”"2.

\section{Sobre la enfermera, continúa:}

"Ella también puede ser descrita como otro brazo del médico o del cirujano. Su función es instrumental, aunque no sólo mecánicamente instrumental. Tal vez en ciertas relaciones ella es casi una colaboradora. Sin embargo, básicamente es el médico quien observa, reflexiona y decide. La enfermera está en sus manos, lleva a cabo sus órdenes. Es convocada como un centinela en casos de emergencia, quien subordina con lealtad su inteligencia a la teoría y las políticas del médico, y secunda sus esfuerzos"”.

\footnotetext{
2 "The physician thinks, decides, and orders; the pharmacist obeys-obeys of course with discretion, intelligence, and skill-yet in the end obeys and does not originate. Pharmacy therefore is an arm added to the medical profession, a specially and distinctly higher form of handicraft, not a profession".

${ }^{3}$ La cita referenciada expresa textualmente: "She, too, may be described as another arm to the physician or surgeon. Her function is instrumental, though not, indeed, just mechanically instrumental. In certain relations, she is perhaps almost a collaborator. Yet, when all is said, it is the physician who observes, reflects, and decides. The trained
}

Aunque hasta aquí la perspectiva de Flexner parece bastante lógica y certera, habida cuenta de la temporalidad en que desarrolla su teoría, podemos encontrar tres problemas en esta concepción, que limitan su aplicabilidad hoy en día. En primer lugar, el desarrollo industrial que contextualiza sus escritos fue concebido desde la noción de división del trabajo en dos componentes: trabajo intelectual y trabajo instrumental (10). Más aún, el desarrollo industrial originó una cultura de trabajo que, a su vez, desarrolló una visión "científica" de la gestión del trabajo, en términos de fragmentación, coordinación, precisión, perfección, exactitud y puntualidad -por mencionar algunos- que paradójicamente causó la fisión de tareas y el surgimiento de nuevas especialidades ${ }^{4}$. Una imagen más realista y actual sobre el trabajo sería una ocupación completa que incluya dos componentes: una esfera intelectual y una esfera instrumental, ambas dimensiones presentes en una misma ocupación.

En segundo lugar, trayendo su teoría al caso específico de la enfermería, los problemas de salud son a menudo tan complejos (12) que la delimitación entre especialidades y profesiones es confusa, por tanto una línea clara de demarcación entre campos disciplinares es prácticamente imposible. Por ejemplo, bajo ciertas condiciones una enfermera debe actuar como médico -la enfermera define problemas de salud, prescribe, trata y evalúa- así como el médico puede actuar como enfermera - alimenta, viste, toma signos vitales, da soporte emocional, etcétera. Lo que quiero subrayar aquí es el hecho de

nurse plays into his hands; carries out his orders; summons him like a sentinel in fresh emergencies; subordinates loyally her intelligence to his theory, to his policy, and [is] effective in precise proportion to her ability thus to second his efforts." Traducción libre.

${ }^{4}$ Estos cambios en las relaciones sociales, en palabras de Dingwall (11), corresponde a la aplicación de los principios tayloristas de racionalización, tales como la delegación del "trabajo sucio" a las ocupaciones subordinadas, modo en que una actividad singular se diferencia y se convierte en un nuevo campo de trabajo. Muchas de las ocupaciones paramédicas de hoy evolucionaron en base a este principio. 
que la demarcación en el terreno de la salud es a menudo meramente legal, sin una división del trabajo intelectual/instrumental inconfundible. De hecho, los trabajadores de salud son conscientes de este tipo de interacción mutua entre roles, la cual tiene lugar en una amplia gama de situaciones.

El tercer problema en concebir una profesión desde una perspectiva estructural es el peso y el orden de los atributos descritos por Flexner, tanto en lo individual como en lo colectivo, en términos de importancia, grado de interrelación, y estabilidad en el tiempo (13). En este sentido, ¿es el altruismo que experimenta la enfermera más importante que la autonomía y el monopolio del que goza un abogado? O dentro de la enfermería misma, ¿es el cuerpo de conocimientos científicos más trascendente que la ética profesional? En una lectura de este tipo, asumo que no todos los rasgos se han desarrollado homogéneamente ni son tratados con igual prioridad por este grupo profesional.

Me resultaría difícil no considerar que la visión de Flexner fue un producto concreto de las ideas del siglo XIX, cuando en la sociedad occidental surge la idea de que las personas tienen control sobre su destino y sobre cada esfera de la vida humana, no solamente en términos filosóficos sino también en aspectos humanos prácticos, como el trabajo y la prosperidad. La incipiente clase media emergente gana creciente atención y se idean formas de mantener los límites objetivamente definidos, no solamente como una abstracción sino como una manera de dar forma a las relaciones y preservar la actividad profesional como un símbolo social de exclusividad.

Por otra parte, en los inicios del siglo XX la medicina, el derecho y el sacerdocio descansaban en tradiciones y prácticas culturales basadas en la autointerpretación, en términos de prestigio y respeto social $(14,15)$, como también en la posición política de la que gozaban. De esta manera podían moldear sus propios grupos profesionales tanto como causar efectos tangibles en el resto de la sociedad, como por ejemplo vigilar y restringir el surgimiento de nuevas profesiones.

Si hay un rasgo que para bien o mal puede hoy arruinar el enfoque estructural, es su metodología. Las observaciones de Flexner, aunque muchos autores estuvieran de acuerdo con sus resultados, se llevaron a cabo casi solamente observando médicos, abogados y sacerdotes, los que todavía hoy representan una concepción dominante y masculina del trabajo (16). En esta visión, campos como la enseñanza o la enfermería son considerados una expresión de amor y compasión, más unido al altruismo que al trabajo 'real', consecuentemente menos posible de ser considerados profesiones. Este hecho implica varias consecuencias para las nuevas categorías ocupacionales como la enfermería, la educación o el trabajo social. En efecto, éstas experimentan dificultades para ser legitimadas formalmente por las autoridades legales. No obstante, sus representantes son rápidos en responder "profesional" cuando son preguntados por el estatus de estas ocupaciones, argumentando el cuerpo teórico, su alegada autonomía en la práctica, el reconocimiento social reflejado en la legislación, y la profundidad y extensión de los estudios. Una vez más estos rasgos representan una falacia. Por ejemplo, si los programas de estudios de todas las ocupaciones manuales, como la de jardinero o vigilante, decidieran extender sus estudios profundizando en cursos científicos -como los efectos químicos de los fertilizantes o los principios de la botánica- y en la abstracción de su contribución a la sociedad -como las consideraciones éticas sobre el acceso a información de la vida privada de quienes habitan un edificio-, todos podrían disputar un estatus profesional. Lo mismo vale para el reconocimiento social, que en el caso ocupaciones emergentes ha sido ampliamente debatido el hecho de que la población general desconoce su verdadero papel en la sociedad contemporánea, por tanto podemos asumir que su aparición en la legislación no es realmente un reconocimiento 
sino el resultado de la habilidad de sus representantes para negociar políticamente con el legislador. También la materialización de aspectos como la autonomía es generalmente parcial, habida cuenta de los esfuerzos de las profesiones clásicas para proteger su reputación y dominio.

Ciertamente, muchas ocupaciones fueron y todavía parecieran estar atrapadas en un cierto "limbo sociopolítico", entre las categorías "profesional" y "no profesional", puesto que el cumplimiento de los rasgos que ofrece el enfoque estructural es más bien una materia de acuerdo o desacuerdo, o una discusión ideológica, pero no un dato realmente objetivo.

\section{Orden social y trabajo profesional en la noción teológica}

En esta sección presento una visión general de la concepción teológica sobre el orden social y cómo ésta ha influido en la noción estructural del trabajo profesional que discutí en el apartado anterior. Con esto fundamento la socialización de enfermería en una lógica corporativa que subraya los aspectos estructurales del trabajo profesional.

Pareciera que la medicina y el derecho son los íconos incuestionables de profesionalismo, tanto que prácticamente no hay estudios sobre trabajo profesional que no tomen como referente a estos dos grupos. Estos antiguos trabajos tienen sus raíces en el modelo teológico medieval sobre trabajo y Estado $(17,18)$ entendido como una compleja estructura analítica del mundo occidental, sostenida hasta bien avanzado el siglo veinte. Esta aproximación tiene su origen en la visión dogmática que eleva al Estado a una categoría de garante exclusivo del derecho universal, el cual define la filosofía política y el sistema de estado corporativo (Corpus $=$ cuerpo). De esta manera el corporativismo -por definición el Cuerpo de Cristo- es traspasado desde el catolicismo a la estructura política del Estado moderno.
En esta escena, los oficios y las profesiones estuvieron en el centro del desarrollo de la comunidad medieval (19). Los propios trabajadores se agrupaban en barrios de acuerdo a sus oficios, disputando la definición y delimitación de su experticia y del territorio que pretendían mantener bajo control.

El estatus profesional significaba la mantención de un particular estado mediante una entidad jurídica legitimada por medio de tal calificación. Podemos todavía encontrar grados académicos que reflejan este principio, tal como el de Profesor de Estado, Diploma de Estado de Doctor en Medicina ${ }^{5}$, etcétera. En este sentido, la agrupación de miembros refleja el sentido corporativo de la noción teológica del orden social y de la administración pública. Así también, una profesión significaba profesar una ideología, hacer de una actividad laboral una opción de vida, por medio de la ordenación que le consagraba como experto en determinada materia, para profesar un ministerio. En este cuadro, la medicina y el derecho fueron las primeras disciplinas no teológicas creadas en el núcleo de la universidad medieval (20).

Ésta es la vía a través de la cual el trabajo pasa a convertirse en un asunto de tal importancia en la vida cívica, tanto como en la vida espiritual. Siguiendo esta doctrina católica, una persona que no trabaja no tiene dignidad para vivir, lo cual llega a reforzarse mediante la integración iglesia-Estado en términos simbólicos de orden y jerarquía. Esta dignidad, no obstante, también refleja las diferencias sociales entre las 'artes liberales' -las que poseen el estatus de profesión-y las 'artes manuales', sin tal estatus. Las artes liberales eran sagradas, intelectuales, teóricas, abstractas. En contraste, las artes manuales eran profanas, empíricas, prácticas, populares. Esta estratificación se apoya, de hecho, en la visión androcéntrica y jerárquica de la

\footnotetext{
5 En Chile esta denominación tiende a desaparecer. Al contrario, es aún común en países en que el Estado tiene una participación importante en la educación.
} 
religión romana, no solamente sobre el trabajo, sino también sobre la vida y la sociedad, cuya influencia se expandió sobre todo el mundo occidental.

Podría contraargumentarse, no obstante, la emergencia de un modelo alternativo sobre el trabajo profesional, más identificado con el mundo anglosajón. En efecto, como resultado de la Reforma en Alemania, y la escisión de Inglaterra del catolicismo surge una perspectiva un tanto diferente sobre orden social y trabajo. En un análisis detallado, no obstante, pueden identificarse los mismos elementos estructurales del catolicismo pero conectados de un modo diferente; por ejemplo, la vocación (vocatio = la voz de dios), puede surgir como un llamado personal sin intermediarios; la actividad laboral, por su parte, apoyada y regulada por una comunidad de iguales, conformando una fraternidad. Éstos son elementos alojados en el núcleo del dogma y del derecho germánico, una concepción völkisch del orden social, en oposición al orden sagrado romano $(21,22)$. Por tanto, este regreso del orden social a los valores del pueblo no parecen haber significado gran cambio en las profesiones.

De cualquier modo, el origen religioso de las profesiones ha tenido un gran impacto en la manera en que crea una dicotomía entre asuntos sagrados y asuntos seculares, o en otras palabras, entre gente con educación y gente sin educación, entre orden y desorden. Desde este punto de vista, la profesión es por definición profesar un conocimiento que es únicamente "conocible" por medio de la fe, de la conversión a una ideología, y por un llamado personal, la vocación. De ahí que los discípulos o aprendices no puedan prepararse por sí mismos sino siguiendo a un maestro que los asista en la aceptación de este llamado y en el desarrollar habilidades, valores y creencias. Este hecho era $-\mathrm{y}$ a mi entender lo sigue siendo- una manera de lograr legitimidad, conocimiento y práctica, pero también una vía por la cual los profesionales buscan exclusividad y singularidad, lo que a la larga termina en la creación de un linaje. De este modo, la comunidad de pares profesionales se convierte en un símbolo social subrayado a través de los ritos de transición, por ejemplo el juramento aún presente en la enfermería y en otras disciplinas de salud, orquestado por la simbología litúrgica y el ceremonial de iniciación. Esto trae consigo la unión de dos componentes: la admisión dentro del linaje y la adopción de un conjunto de reglas que los mantiene consagrados dentro del servicio.

Mientras que este análisis es relevante en la arena político-teológica, ¿cuáles son las implicancias para la ocupación de enfermería? Parece incuestionable que esta noción de trabajo está todavía muy presente en características observables de la enfermería, por ejemplo los uniformes, que de alguna manera u otra evocan los hábitos religiosos. También en sus íconos, como la lámpara de aceite que emite una flama, universalmente aceptada como símbolo de iluminación y conocimiento, y aun en la autointerpretación de las enfermeras que se traduce en mayor o menor medida en expectativas mutuas de sacrificio y rigor. Por otra parte, es un hecho el que muchas escuelas de enfermería y también los primeros hospitales contemporáneos fueron constituidos en torno a la visión de Nightingale sobre el trabajo femenino, y de las órdenes religiosas ligadas a la salubridad y la caridad. No es en un vacío donde las enfermeras son socializadas, sino en un marco implícito de matriz cristiana, por tanto en la idea de vocación y abnegación más que en la idea de profesionalismo como podría entenderse en otras ocupaciones.

Una vez más, aunque inspiradora para muchos especialmente en discusiones sobre el ethos de la enfermería, esta noción teológica de profesionalismo no permite responder a cuestiones sobre si la enfermería es o no una profesión, sobre todo en sociedades que experimentan crecientes niveles de laicización, y en que valores de matriz cristiana tienden a ser influidos por otras categorías sociales. 
Un análisis de este tipo, aunque no tan infrecuente en las escuelas de enfermería, puede ser errático al enfocarse sólo en aspectos históricos más que en las fuerzas que moldean las profesiones de hoy en día, y cómo éstas se conectan unas a otras influyéndose mutuamente.

\section{Una teoría sistémica para el desarrollo de enfermería}

La visión teológica y la noción industrial de trabajo abordadas en las secciones anteriores pueden parecer poco relacionadas con cómo opera el trabajo en la sociedades de hoy. No obstante, al hablar de profesiones en nuestro tiempo, me parece claro que la discusión se dirige instintivamente a argumentar sobre cómo la estructura de la enfermería se construye sobre los valores transpuestos desde la escena teológica: la vocación, la preparación guiada bajo tutela experta y el juramento de profesar bajo un código que integra ciertos criterios de buena práctica.

Por sobre todo, es la forma institucional de la enfermería, su estructura interna, lo que parece ser argumentado y férreamente defendido en el marco de las prerrogativas profesionales: la existencia de asociaciones profesionales, la autorregulación de la práctica, la formación abstracta, la autonomía y monopolio del ejercicio, por nombrar sólo algunas. Esta aproximación, como he venido discutiendo, deja de considerar las implicancias recíprocas que las ocupaciones tienen entre sí, la competición interprofesional, la delegación sistemática de tareas indeseables en el fenómeno de creación de ocupaciones nuevas, procesos que se han dado casi de manera natural en la diversificación de las especialidades, como por ejemplo escisión, fusión y fisión de las ocupaciones en la medida que evolucionan (11).

Un análisis sistémico (23-27) de la enfermería necesitaría tomar en cuenta estos fenómenos de diversificación, como también los aspectos que definen, constriñen o expanden los límites de la práctica en esta escena sistémica. Como en toda ecología, es fundado considerar que unas ocupaciones son más dominantes que otras, que compiten por un dominio y por nutrientes, que se desarrollan de manera muy diversa, que despliegan mecanismos de supervivencia y adaptación, que pueden actuar en relaciones simbióticas, o que pueden sufrir mutaciones, incluso extinguirse.

Como advierto en los escritos influyentes de la literatura de enfermería (6-8), la versión teológica y la perspectiva estructural han prevalecido en el análisis. A mi entender, explicar una profesión por esta vía equivaldría a hacer inteligible al ser humano sólo desde un punto de vista anatómico, la descripción de su estructura ósea, de los nervios y músculos que permiten el movimiento, pero no el funcionamiento de estos tejidos, el desarrollo de la personalidad ni la posición en la vida en sociedad o en el sistema ecológico.

Lo que deseo argumentar aquí es que si bien el cómo una ocupación se estructura es relevante como punto de partida, el estatus profesional no puede ser tomado como una verdad evidente en sí misma, dogmática. En otras palabras, un marco interpretativo sistémico requeriría, en su sentido más profundo, examinar lo que la enfermería hace (28-29), no cómo está estructurada para hacer lo que hace. Se trata de un análisis cercano al sistema ocupacional completo, a sus intrincados mecanismos de interconexión y no a cada pieza por separado como se ha hecho hasta ahora.

En este sentido, ¿cuál es la pieza que la enfermería juega en el puzle completo de la esfera del trabajo?, ¿cuáles son los modos sociales de relaciones recíprocas con las otras piezas?, ¿de qué manera ideas del postfeminismo y de las nuevas masculinidades modelan hoy las relaciones entre médicos y enfermeras?, ¿qué papel juegan las interacciones basadas en clases sociales en esa ecología?, ¿hasta qué punto aspectos como autonomía y monopolio son congruentes en la prácti- 
ca con las construcciones discursivas?, ¿qué se puede concluir en cuanto a la etnicidad $\mathrm{y}$ alcance de un proyecto profesionalizante considerando las dramáticas diferencias en desarrollo que la enfermería experimenta comparativamente entre países, regiones y continentes? (30-32).

Estos aspectos, si bien algunos han sido analizados aisladamente, necesitan un examen más cercano en una dimensión de simultaneidad. Un análisis sistémico tiene mucho más para ofrecer que el simple estudio estructural de la enfermería, el cual ha conducido erráticamente a esgrimir estatus profesional en base al aparato estructural logrado casi únicamente por la enfermería del mundo anglosajón, donde se produce mayoritariamente la teorización disciplinar. La resultante debería esclarecer sobre la posición de la enfermería en su ecosistema, sus relaciones de mutualidad, competencia por territorio, patrones de dominación, potencial de extinción, y entonces preguntarse si el comportamiento podría ser considerado profesional.

\section{CONSIDERACIONES FINALES}

En este artículo he intentado plantear que las fuerzas externas, desde el "ecosistema", pueden más potentemente incentivar nuevas preguntas y nuevas maneras de entender una actividad ocupacional y sus prerrogativas de estatus profesional $(6,7)$.

Sin una teoría sistémica de desarrollo (2327) como la sugerida aquí, el estatus profesional de la enfermería permanecerá siendo un tópico activamente defendido, sin embargo la discusión se basará fundamentalmente en el sentido persuasivo del concepto y esgrimiendo logros estructurales, sin considerar técnicamente la enfermería en la escena ecológica. Mediante el uso de la persuasión y argumentando aspectos estructurales las prerrogativas profesionales permanecerán focalizadas en los deseos de reconocimiento y reivindicación. $\mathrm{O}$ a lo sumo habrá intentos por demostrar de distintas maneras la existencia de un patrón común de desarrollo estructural de todas las profesiones, un proceso de profesionalización $(33,34)$, y de potenciar la academicidad de la enfermería (35, 36), que una vez más redundarán en asuntos estructurales.

Con todo, en modo alguno mi reflexión debería conducir a detener los proyectos de desarrollo para la enfermería como ocupación, más bien debería esclarecer las limitaciones en su formulación y estimular el desarrollo de marcos interpretativos que renueven y fortalezcan tanto su construcción teórica como suavizar tensiones internas del ámbito de la práctica. Hay fenómenos de cambio social que ponen a la enfermería en una posición privilegiada para idear y llevar a cabo estas modificaciones, como a plantearse nuevas preguntas, sistémicamente orientadas, sobre su desarrollo profesional futuro.

\section{AGRADECIMIENTOS}

A la Comisión Europea que ha apoyado este proyecto a través del esquema de cooperación y mobilidad internacional en educación superior "EMECW".

Al Sr. David Benton, Chief Executive Officer del International Council of Nurses, por proporcionarme información de interés. Al Sr. Bernardo Alarcón P., abogado, en traducir el original en inglés. También mi gratitud para los revisores anónimos, cuyos comentarios contribuyeron a enriquecer la primera versión de mi manuscrito.

\section{REFERENCIAS}

1. Flexner A. Is social work a profession? En Proceedings of the National Conference 
of Charities and Correction. Chicago: Hildemann; 1915. p. 577-90.

2. Richmond ME. The Social Case Worker's task. En Proceedings of the National Conference of Social Work. Chicago: Rogers \& Hall Co; 1917.

3. Cook WW. Improvement of legal education of standards for admission to the bar. Me L Rev. 1918. 11(3): 92.

4. LeRosginol JE. Dr. Flexner on university training for business. J Bus Univ Chic. 1931; 4(3): 127-137.

5. Morrill JL. Is There a Place For Instruction in Journalism? Journal Q. 1938; 15: 28-34.

6. Bixler G, Bixler R. The professional status of nursing. Am J Nurs. 1945; 45(9): 730735.

7. Bixler G, Bixler R. The professional status of nursing. Am J Nurs. 1959; 59(8): 11421147.

8. Leddy S, Pepper JM. Conceptual Bases of Professional Nursing. 4ta ed. Philadelphia: Lippincott; 1998. 477 p.

9. Bourdieu P. La distinction. Critique sociale du jugement. París: Les Éditions de Minuit; $1979.672 \mathrm{p}$.

10. Veblen T. The Theory of the Leisure Class. New York: Modern Library; 1934. 404 p.

11. Dingwall R. Essays on Professions. Aldershot: Ashgate; 2008. 195 p.

12. Holmes DR. Confronting complex public health Problems: the development of interdisciplinary research. J Public Health Policy. 1981; 2(4): 361-381.

13. Brante T. Sociological approaches to the Professions. Acta Sociol. 1988; 31(2): 119-142.

14. Burnham J. American Medicine's Golden Age: What happened to it? Science. 1988; 215(4539): 474-79.

15. Gordon RW. The American legal profession, 1870-2000. En: Grossberg M, Tomlins $\mathrm{C}$, eds. The Cambridge History of Law in America. Vol 3. Cambridge: Cambridge University Press; 2008. p. 73-126.

16. Adkins L. Gendered Work: Sexuality, Fa- mily and the Labour Market. Milton Keynes and Philadelphia: Open University Press; 1995.

17. Bouckaert B. The Roots of our Liberties: On the Rise of Civil Society in the Medieval West. NPPE. 2007; 3(2): 139-184.

18. Bourdin B. La genèse théologico-politique de l'Etat moderne. Paris: Les Presses Universitaires de France; 2004. 280 p.

19. Dubar C, Tripier P. Sociologie des professions. Paris: A. Colin; 1998. 256 p.

20. Carr-Saunders AM, Wilson PA. The Professions. Oxford: Clarendon Press; 1933. $536 \mathrm{p}$.

21. Hahn HJ. German thought and culture. From the Holy Roman Empire to the present day. Manchester: Manchester University Press; 1995. $251 \mathrm{p}$.

22. Thornhill CJ. German Political Philosophy: The Metaphysics of Law. New York: Routledge; 2006. 412 p.

23. Abbott A. Status and status strain in the professions. AJS. 1981; 86(4): 819-835.

24. Abbott A. The System of Professions: An Essay on the Division of Expert Labor. Chicago: The University of Chicago Press; 1988. 452 p.

25. Abbott A. Things on boundaries. Soc Res. 1995; 62(4): 857-882.

26. Abbott A. Chaos of Disciplines. Chicago: The University Chicago Press; 2002. 248 p.

27. Abbott A. Varieties of Ignorance. Am Soc. 2010; 41: 174-189.

28. Allen D. What do you do at work? Profession building and doing nursing. Int Nurs Rev. 2007; 54(1): 41-48.

29. Allen D. The nursing-medical boundary: a negotiated order? Sociol Health Illn. 1997; 19(4): 498-520.

30. Sciulli D. Continental sociology of professions today: conceptual contributions. Curr Sociol. 2005; 53(6): 915-942.

31. Sciulli D. Professions in civil society and the state: invariant foundations and consequences. Leiden, The Netherlands: Brill; 2009. 490 p. 
32. Siegrist H. Public office or free profession? German attorneys in the Nineteenth and early Twentieth century. En: Cocks G, Jarausch CH, eds. German Professions, 1800-1950. New York: Oxford University Press; 1990. p. 46-65.

33. Watson TJ. The professionalization process: a critical note. Sociol Rev. 1976; 24(3): 599-608.

34. Dingwall R. The inevitability of professions? En: Currie G, Ford J, Harding N, Learmonth M, eds. Making public servi- ces management critical. London: Routledge; 2009. p. 71-85.

35. Fealy GM, McNamara MS. A discourse analysis of debates surrounding the entry of nursing into higher education in Ireland. Int J Nurs Stud. 2007; 44(7): 11871195.

36. Ayala RA, Fealy GM, Vanderstraten R, Bracke P. Academisation of nursing: An ethnography of social transformations in Chile. Int J Nurs Stud. En prensa 2013. 